\title{
A GENERIC DATA MODEL FOR SCHEMA-DRIVEN DESIGN IN INFORMATION RETRIEVAL APPLICATIONS
}

Hany Azzam, and Thomas Roelleke

\{hany, thor\}@eecs.qmul.ac.uk

\section{INTRODUCTION}

- Large-scale knowledge bases can be automatically generated from high-quality knowledge source such as Wikipedia and other semantically explicit data repositories

- These knowledge bases are leveraged by IR application developers to develop more semanticallyaware retrieval systems

- Developing new applications or incorporating new data formats usually requires "reimplementing APIs, introducing new query languages, and even introducing new indexing and storage structures"

\section{Motivation}

- How diverse applications and data formats can be supported by a single unifying framework?

- How techniques developed for a particular data ormat such as text can be transferred/extended to other data formats?

\section{Contributions}

- A generic data model is proposed that represent facts (e.g. objects and their relationships) and content knowledge (e.g. text in documents) in one congruent data model and can be used to transfer text retrieval models such as language modelling to classification, relationship and attribute-based retrieval models

- A simplified design that distinguishes between three modelling layers: basic, structural and semantic, which can then be used to derive application-independent and application-specific schemas

\section{REFERENCES}

[1] R. Cornacchia and A . de Vries. A parameterised search system. In ECIR, 2007 .

[2] N. Fuhr. Towards data abstraction in networked information retrieval systems. IP\&SM, 35(2):101-119, 1999.

3] D. Hiemstra and V. Mihajlovic. A database approach to information retrieval: The remarkable relationship between language models and region models. CTIT Technical Report, 2010.

4] C. Meghini, F. Sebastiani, U. Straccia, and C. Thanos. A model of information retrieval based
on a terminological logic. In SIGIR, 1993 .

\section{The Data Model}

- The data model, the Probabilistic Object-Oriented Content Model (POOCM), combines probability theory, object-oriented modelling and content-oriented modelling

- It consists of classifications, relationships, attributes and terms, which are all referred to as propositions

$<$ movie id="329191" title="Gladiator" year="2000" genre="action">

$<$ actor $>$ Russell Crowe $<$ /actor $>$

$<$ team $>$ Ridley Scott $</$ team $>$

$<$ plot $>$ When a $[$ ARG1 general]

is [TARGET betrayed by ] a

$[$ ARG0 prince] ... </plot $>$

\# Term 'gladiator' in movie_329191

0.5 gladiator(movie_329191)

\# Classification 'russell_crowe is an actor' in imdb

0.7 actor(russell crowe, imdb);

\# Classification 'maximus is a general' in movie_329191 general(maximus, movie_329191);

\# Relationship 'russell_crowe playsRoleOf

\# maximus' in movie_329191

playsRoleOf(russell_crowe, maximus, movie_329191);

\# Attribute 'movie_329191 has genre action' in imd genre(movie_329191, action, imdb)

\section{Modelling LAYers}

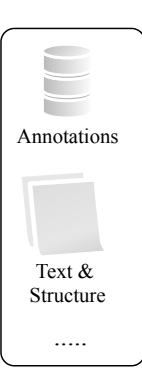

Data

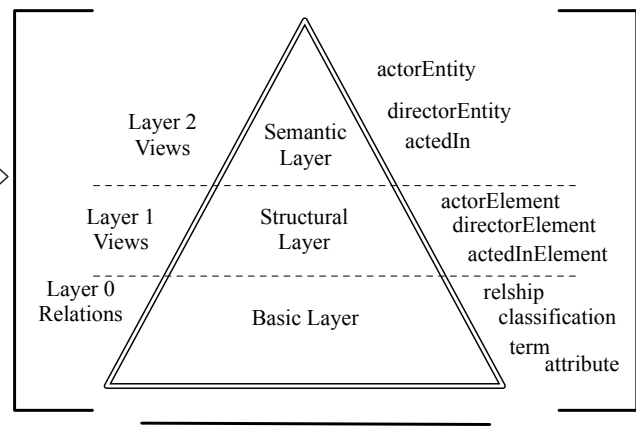

The POOCM Data Model

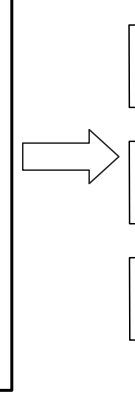
Query

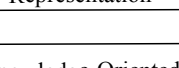

Knowledge-Oriented

Consolidated Data

Representation

Application
- Application-independent and application-specific schemas can be instantiated:

- Layer-0 is the basic, application-independent layer

- Layer-1 is the element-based layer. It contains rules that can derive structural predicates from the L0. These rules can lift the basic classifications and attributes into structural classifications and attributes

- Layer-2 is the entity-based layer. It contain rules that derive semantic classification and relationships. For example, the rules extract objects by combining structural information about element types and their attributes

- Note that L2 relation names bear a meaning, L1 relation names indicate the type of the context, and $\mathrm{L} 0$ relation names reflect classifications and relationships

- The layers form an abstraction hierarchy that helps to achieve data independence. Any data format (e.g. XML, RDF, text) can be represented

- Indexing and processing strategies developed for one type of an application become transferable

- Explicitly stating how the lavers are related can impact the modelling of probability estimations and aggregations

\section{Probability Estimation}

- As probabilities are an inherent component of the data model, probabilistic relations used to develop retrieval models can be estimated for each of the layers

- L1 consists of relations tailored to modelling the structure of data, such as relations for contextbased segmentations, e.g. term_doc(Term,Doc) to index documents, and term $\_$sec(Term,Sec) to index sections.

- L2 comprises of relations reflecting the $s e$ mantics of the data (semantic lifting of L1 leads to L2 relations). For instance, "actedIn(Actor,Movie,Context)" is L2.

- $P_{\mathrm{VF}}(t \mid i)$ : Value Frequency-based probability of term $t$ derived from an index $i$ such a "term(Term, Doc)" where the occurrence in documents (values) is the evidence.

- $P_{\mathrm{TF}}(t \mid i)$ : Tuple Frequency-based probability of term $t$ derived from an index $i$ where the occurrence in locations (tuples) is the evidence.

- $P_{\mathrm{IVF}}(t \mid i): \quad \begin{gathered}\text { Inverse Value Frequency } \\ \text { based }\end{gathered}$ based (IVF) probability of term $t$ e.g. $\quad-\log P_{\mathrm{VF}}(t \mid i) / \max \left(\left\{-\log P_{\mathrm{VF}}\left(t^{\prime} \mid i\right)\right\}\right)$ retrieval InvActorFreq=IVF

\section{BenEFits}

- The data model allows the handling of the physical data structures to remain decoupled from the rest of the system design and the retrieval models

- The object-oriented and content-oriented concepts provide the ability to instantiate retrieval models consisting of term, classification, relationship and attribute propositions

- This schema-driven approach helps to quickly incorporate different data formats into a standard representation and provides models that support constraint-checking and ranking with respect to these data formats.

- Overall, the data model can improve the develop ment process of IR applications 